\title{
KVN Calibrator Survey (KVNCS) and VLBI follow-ups
}

Jeong Ae Lee*

Korea Astronomy and Space science Institute and University of Science and Technology

E-mail: ialeedkasi.re.kn

\section{Bong Won Sohn}

Korea Astronomy and Space science Institute, University of Science and Technology

E-mail: bwsohnakasi,re.kn

\section{Taehyun Jung}

Korea Astronomy and Space science Institute

E-mail: thjungekasi.re.kn

\section{Do-Young Byun}

Korea Astronomy and Space science Institute

E-mail: bdydkasi.re.kr

\section{Jeewon Lee}

Korea Astronomy and Space science Institute and Kyunghee University

E-mail: jwlee78dkasi.re.kr

We have performed Korean VLBI Network Calibrator Survey (KVNCS) to obtain the sufficient VLBI phase calibrators at K band. The samples were taken from VLBA Calibrator Survey (VCS). We measured their single-dish flux density for 6 epochs from 2009 to 2011 with 21-m KVN antennas at $\mathrm{K}$ and $\mathrm{Q}$ bands simultaneously. The flux density of 1533 and 553 sources were obtained at $\mathrm{K}$ and $\mathrm{Q}$ band respectively. Their distribution covered almost whole sky with declination $\geq$ $-32^{\circ} .5$ and unbiased. About 1000 sources were not previously detected at K band with VLBI. We tried to search for VLBI fringes of 556 sources which had the single-dish flux density $\geq 200 \mathrm{mJy}$ with KVN. Detection rate was about $80 \%$ on one baseline. More detailed analysis are processing.

11 th European VLBI Network Symposium \& Users Meeting,

October 9-12, 2012

Bordeaux, France

\footnotetext{
* Speaker.
} 


\section{Introduction}

In order to correct the unpredictable path-length delay, phase-referencing technique (PR) has been suggested (Beasley and Conway 1995, references therein). The phase of target can be compensated by one of calibrator with this technique. Fast antenna switching (Holdaway et al. 1995, references therein) is typical PR technique, however, it needs adjacent phase calibrators. Although PR is useful to modify the phase, it still needs faster moving antenna and simultaneous multifrequency technique. Korean VLBI Network (KVN) has equipped the optimum and unique system. 22, 43, 86 and $129 \mathrm{GHz}$ simultaneous multi-frequency observing system with full polarization (Han et al. 2008). However, phase calibrators are still insufficient within coherence scales. KVN calibrator survey (KVNCS) aimed the dense distribution of calibrators with declination $\geq-32.5$ at $\mathrm{K}$ band. In order to confirm detectability at KVN, the single-dish observations were performed at $\mathrm{K}$ and $\mathrm{Q}$ bands simultaneously. And then, their VLBI fringes were searched for checking a validity as the candidates of VLBI phase calibrators.

\section{Single-dish observation (KVNCS1)}

To measure the single-dish flux density of the samples, we carried out the observation with KVN Yonsei or Ulsan antennas at K and Q bands simultaneously.

\subsection{Observation}

The samples were taken from VLBA Calibrator Survey (VCS, Beasley et al. 2002; Fomalont et al. 2003; Petrov et al. 2005; Petrov et al. 2006; Kovalev et al. 2007; Petrov et al. 2008). Their extrapolated total flux were calculated from the total flux at S and X bands (Sohn et al. in prep.). 2503 sources were selected and their extrapolated flux density were greater than $100 \mathrm{mJy}$ with declination $\geq-32^{\circ} .5$ at $\mathrm{K}$ band. The observations were performed at $21.7(\mathrm{~K})$ and $42.4(\mathrm{Q})$ bands in Dec. 2009 - Mar. 2011 at KVN Yonsei or Ulsan observatories. Cross-scan mode was used for detecting gaussian peak flux density. To estimate the more precise flux density of sources, 3C 286 was observed regularly as the primary flux calibrator. Its flux had been measured using a brightness model of Mars at KVN Yonsei observatory (Sohn, in prep.).

\subsection{Results and Discussion}

We measured the flux density of 1533 and 553 sources with $\geq 3 \sigma$ detection at K and Q bands respectively. Table 1 shows the resulted number of sources. Their coverage on the celestial sphere shows in Figure 1. These sources distribute on the unbiased directions. More analysis are going to present in forthcoming paper (Lee et al. in prep).

\section{K-band KVN Fringe Survey (KVNCS2)}

1147 of 1533 sources were not previously detected at K band with VLBI. In order to confirm their VLBI fringes, we selected 556 sources which were KVNCS1 flux density $\geq 200$ mJy. 


\subsection{Observation and Data reduction}

Observations were performed on Feb. 2, Apr. 4, 5 and May 22, 2012 at 22.700GHz with KVN. Integration time of each source was 120 s. A scheduling was composed by sur_sked, minimizing the slewing time. Every sources was observed with Sky/Vain calibration and we executed sky-dipping mode every an hour. The recording used Mark-5B in 16 intermediate sub-bands of $16 \mathrm{MHz}$. The data were correlated with DiFX-2 correlator (Deller et al. 2011). We used data analysis using AIPS for searching their fringes.

\subsection{Results and Discussion}

We detected VLBI fringes from about $80 \%$ sources on 1 baseline. The detection rate on 3 baselines was about $67 \%$. Figure 2 shows the spatial distribution of preliminary detected sources. Detection criteria was $>5$ signal to noise ratio.

\section{Summary}

This study was performed to obtain sufficient candidates as VLBI phase calibrators. We measured the flux density of 1533 and 556 sources at K and Q bands respectively. Their spatial distribution shows that the sources randomly laid on. The possibility as VLBI phase calibrators will discuss in forthcoming paper (Lee et al. in prep.). Moreover, in order to search for VLBI fringes of the sources which were not previously detected at K band with VLBI, we observed 556 sources at $\mathrm{K}$ band with KVN. The detection rate were about $80 \%$ on one baseline. More detailed analysis of this fringe survey will be followed continuously.

\section{Acknowledgments}

We are thankful that the registration fee of 11th EVN Symposium for presenting this study was supported by Radionet 3 .

\section{References}

[1] A.J. Beasley and J.E. Conway 1995, Very Long Baseline Interferometry and the VLBANRAO Workshop No. 22, ASPC 82327

[2] H.A. Holdaway et al. 1995, Fast Switching Phase Calibration: Effectiveness at Mauna Kea an Chajnantor, ALMA memo series 139

[3] A.J. Beasley et al. 2002, The VLBA Calibrator Survey - VCS1, ApJS 14113

[4] E.B. Fomalont et al. 2003, The second VLBA Calibrator Survey - VCS2, AJ 126 2562-2566

[5] L. Petrov et al. 2005, The third VLBA Calibrator Survey - VCS3, AJ 1291163

[6] L. Petrov et al. 2006, The fourth VLBA Calibrator Survey - VCS4, AJ 1311872

[7] Y.Y. Kovalev et al. 2007, The fifth VLBA Calibrator Survey - VCS5, AJ 1331236

[8] L. Petrov et al. 2008, The sixth VLBA Calibrator Survey - VCS6, AJ 126

[9] S.-T. Han et al. 2008, Millimeter-wave Receiver Optics for Korean VLBI Network, IJIMW 2969 
[10] A. Deller et al. 2011, DiFX2: A more flexible, efficient, robust and powerful software correlator, PASP 123275
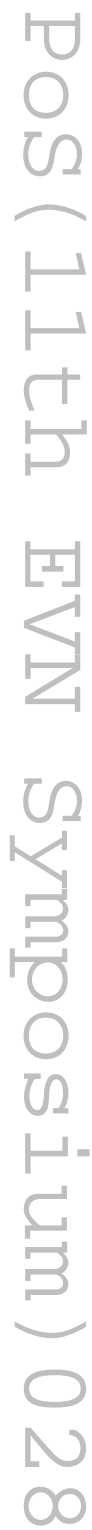


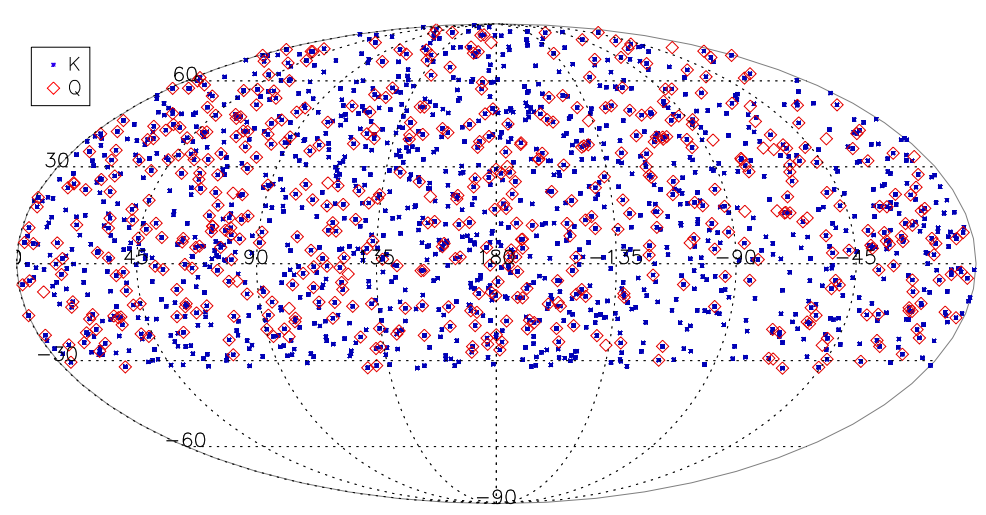

Figure 1: The distribution of 1533 sources drawn by Mollweide projection. Blue $*$ is the sources at $\mathrm{K}$ band and red $\diamond$ at Q band. Abscissa is right ascension [degree] and ordinate is declination [degree].

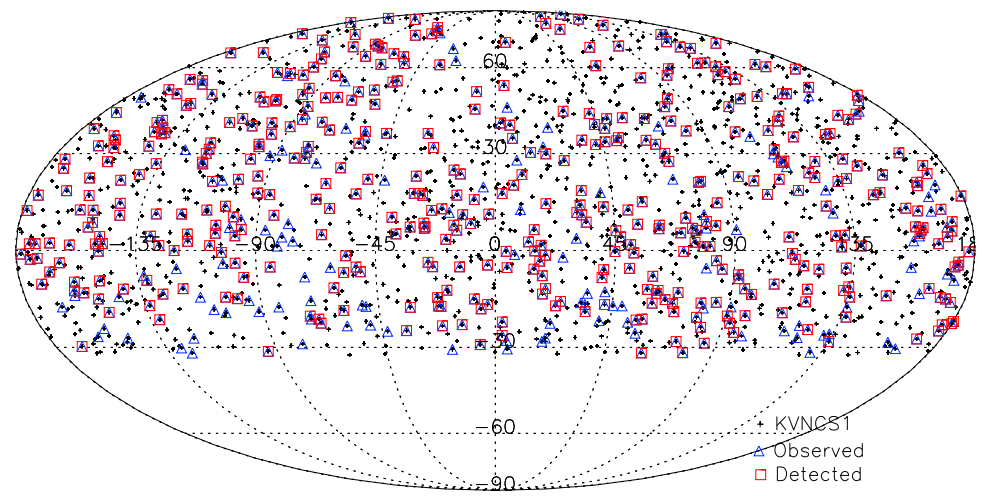

Figure 2: The result of VLBI fringe detection at K band. + is $1533 \mathrm{KVNCS} 1$ sources and blue $\triangle$ is 556 observed sources. Red empty $\square$ are 444 detected sources on 1 baseline. Abscissa is right ascension [degree] and ordinate is declination [degree]. 


\begin{tabular}{|c|c|c|c|c|c|}
\hline Epoch & Date & Observatory & $\begin{array}{c}\text { On-source } \\
\text { integration time }[\mathrm{s}]\end{array}$ & $\begin{array}{l}\text { Observed } \\
\text { [number] }\end{array}$ & $\begin{array}{c}3 \sigma \text { detection } \\
\mathrm{K}, \mathrm{Q} \text { [number] }\end{array}$ \\
\hline $\mathrm{v} 1.0 .1$ & Dec. 2009 & $\mathrm{KYS}^{*}$ & $40(\mathrm{~K}), 20(\mathrm{Q})$ & 595 & 312,81 \\
\hline v1.1.1 & Feb. 2010 & KYS & $40(\mathrm{~K}), 20(\mathrm{Q})$ & & 336,44 \\
\hline v1.1.2 & Mar. 2010 & KYS & $40(\mathrm{~K}), 20(\mathrm{Q})$ & & \\
\hline v1.1.3 & May 2010 & KYS & $40(\mathrm{~K}), 20(\mathrm{Q})$ & & \\
\hline $\mathrm{v} 1.2 .1$ & June 2010 & KYS & $80(\mathrm{~K}), 40(\mathrm{Q})$ & 396 & 161,17 \\
\hline $\mathrm{v} 1.2 .2$ & Dec. 2010 & $\mathrm{KUS}^{\dagger}$ & $80(\mathrm{~K}), 40(\mathrm{Q})$ & 670 & 467,106 \\
\hline v1.3.1 & Jan. 2011 & KUS & $160(\mathrm{~K}), 80(\mathrm{Q})$ & 573 & 407,60 \\
\hline $\mathrm{v} 1.3 .2$ & Mar. 2011 & KYS & $160(\mathrm{~K}), 80(\mathrm{Q})$ & 182 & 46,98 \\
\hline
\end{tabular}

Table 1: The resulted number of sources of KVN Calibrator Survey 1 (KVNCS1) 